\title{
PLANEJAMENTO FATORIAL: UMA FERRAMENTA ESTATÍSTICA VALIOSA PARA A DEFINIÇÃO DE PARÂMETROS EXPERIMENTAIS EMPREGADOS NA PESQUISA CIENTÍFICA.
}

\author{
FACTORIAL DESING: A VALUABLE STATISTIC TOOL TO DEFINE \\ EXPERIMENTAL PARAMETERS APPLIED IN SCIENTIFIC RESEARCH
}

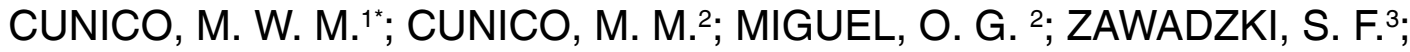 \\ PERALTA-ZAMORA, P. ${ }^{3}$; VOLPATO, N. ${ }^{1}$
}

\begin{abstract}
'Departamento Acadêmico de Mecânica, Núcleo de Prototipagem e Ferramental - NUFER, Universidade Tecnológica Federal do Paraná. Av. Sete de Setembro, n 3165, CEP: 80230-901 -, Curitiba-Paraná. *E-mail: marloncunico@yahoo.com.br

${ }^{2}$ Departamento de Farmácia, Laboratório de Fitoquímica, Universidade Federal do Paraná, Av. Prefeito Lothário Meissner, n 632, CEP: 80210170 - Jardim Botânico, Curitiba-Paraná.

${ }^{3}$ Departamento de Química, Laboratório de Analítica, Universidade Federal do Paraná, Av. Centro Politécnico, CP: 19081, CEP: 81531-990 - Jardim das Américas, Curitiba-Paraná.

${ }^{4}$ Departamento de Química, Laboratório de Polímeros, Universidade Federal do Paraná, Centro Politécnico, CP: 19081, CEP: 81531-990 - Jardim das Américas, Curitiba-Paraná.
\end{abstract}

RECEBIDO: 05/02/08 ACEITO: 01/03/08

\begin{abstract}
RESUMO:
Este trabalho descreve a importância e a conveniência dos procedimentos multivariados de otimização experimental, destacando as vantagens e aplicações dos sistemas de planejamento fatorial que permitem avaliar simultaneamente o efeito de um grande número de variáveis, a partir de um reduzido número de experimentos. Por meio de um exemplo prático é apresentada uma discussão do efeito da interação entre as variáveis e o estabelecimento de ótimos verdadeiros na otimização de parâmetros experimentais envolvidos na fotopolimerização do metacrilato de metila (MMA) comercial, na forma de oligômero, denominado "cola BERKEL", por radiação UV. Aspectos relativos ao desenho experimental e à interpretação de resultados também são apresentados.
\end{abstract}

PALAVRAS-CHAVE: Planejamento fatorial; análise multivariada; fotopolimerização; metacrilato de metila (MMA).

\begin{abstract}
:
This paper describes both the value and the role of multivariated methods of experimental procedure in scientific research. It is highlighted the advantages of factorial design systems which let simulate simultaneously several variables concerned a studied system, reducing the number of experiments of the research. The impact of these variables on the photopolymerization system is discussed upon the photocure of oligo(methyl methacrylate) (MMA), commercially called Berkel glue B 20, initiated by an UV light source. As feedback of the system it was measured the quantity of oligomer converted to polymer (in weight), finding the polymerization rate (in percent). Aspects related to the experimental design and the interpretations of results are also presented.
\end{abstract}

KEY-WORDS: Factorial design; multivariate analysis; photopolymerization; methyl methacrylate (MMA). 


\section{INTRODUÇÃO}

Nos dias atuais, em decorrência das necessidades da sociedade moderna, a pesquisa científica tem promovido grandes avanços em todos os campos da ciência, gerando uma gama crescente de dados e informações, sendo que para a devida exploração e o correto entendimento, a aplicação de ferramentas estatísticas torna-se indispensável (PEREIRA-FILHO et al., 2002).

Diante do exposto e levando em consideração que no Brasil, os recursos financeiros e laboratoriais encontram-se escassos em decorrência das restrições de verbas para a aquisição de equipamentos e materiais de consumo, uma reflexão deve ser realizada no início de cada pesquisa científica, para a definição das condições experimentais, que permitam a aquisição de resultados confiáveis, já que a otimização de parâmetros experimentais de relevância é considerada como uma das etapas mais críticas do trabalho científico, principalmente daqueles que têm por objetivo o desenvolvimento de processos tecnológicos aplicáveis em grande escala (PERALTA-ZAMORA et al., 2005).

Geralmente, a otimização de variáveis experimentais é realizada por meio de procedimentos que avaliam o efeito de uma variável por vez (univariado), apresentando desvantagens tais como o tempo gasto para otimização e a falta de avaliação acerca das interações entre as variáveis que afetam o processo em estudo. De acordo com BRASIL et al. (2007), estas desvantagens resultam numa otimização ineficiente, impedindo o rápido estabelecimento de ótimos verdadeiros, os quais são atingidos pelo emprego de sistemas multivariados (BRASIL et al., 2007).

Dentre os diversos tipos de planejamento experimental, os sistemas de planejamento fatorial destacam-se pois permitem avaliar simultaneamente o efeito de um grande número de variáveis, a partir de um número reduzido de ensaios experimentais, quando comparados aos processos univariados (PERALTA-ZAMORA et al., 2005). Além disso, muitos pesquisadores não contam com uma metodologia de planejamento experimental que seja ao mesmo tempo útil e simples, e estes em geral, apresentam dificuldades para escolher um modelo físicomatemático que represente de maneira adequada os fenômenos que se deseja estudar. Diante do exposto, o objetivo do presente trabalho foi fornecer o conhecimento teórico e prático da metodologia de planejamento fatorial como ferramenta estatística para avaliação e otimização de parâmetros envolvidos numa pesquisa científica experimental envolvendo, como exemplo prático, a polimerização fotoquímica do metacrilato de metila (MMA) com a posterior obtenção do poli(metacrilato de metila), PMMA.

\section{PLANEJAMENTO FATORIAL}

\subsection{CONCEITOS GERAIS DE EXPERIMENTAÇÃO}

Para melhor entendimento dos fundamentos do planejamento fatorial é necessário ter um bom domínio de alguns conhecimentos básicos (CHEMKEYS, 2007, descritos a seguir.

- Fator: cada variável do sistema em estudo.

- Nível: condições de operação dos fatores de controle investigadas nos experimentos. Geralmente são identificados por nível baixo (-) e nível alto (+).

- Modelo estatístico: modelo do tipo $y=b_{0}+b_{1} x_{1}$ sendo $b_{0}, b_{1} \ldots, b_{n}$ efeitos de cada fator na resposta.

- Efeito: mudança ocorrida na resposta quando se muda do nível baixo (-) para o nível alto $(+)$. 
efeito $=\bar{R}_{+}-\bar{R}_{-}$

sendo

$\overline{\mathrm{R}}_{+}=$média dos valores de resposta obtidos com valores altos (+) do fator

$\overline{\mathrm{R}}_{-}=$média dos valores de resposta obtidos com valores baixos (-) do fator

- Efeito principal: é a diferença média observada na reposta quando se muda o nível do fator de controle investigado.

Matematicamente o efeito principal pode ser representado por:

Efeito Principal $=2\left(\sum y^{+}-\sum y^{-}\right)\left(b^{a}\right)$

sendo

$\mathrm{y}=\mathrm{a}$ média dos efeitos individuais da medida, (+) e (-) corresponde ao nível alto e nível baixo,

$b^{a}=$ ao número total de experimentos do planejamento.

- Efeitos de interação: é a metade da diferença entre os efeitos principais de um fator nos níveis de outro fator. Os valores de interação entre os fatores também podem ser calculados. Para tal, o efeito de interação entre as variáveis é obtido levando-se em consideração os sinais já atribuídos às variáveis envolvidas, como se fosse uma operação matemática de multiplicação.

- Erro padrão para os efeitos: erro na determinação dos efeitos. É o mesmo, tanto para os efeitos principais quanto para os de interação, e pode ser calculado por meio de equação.

$$
\text { Erro Padrão do Efeito }=\frac{\mathrm{s}}{\sqrt{2^{k-1}}}
$$

sendo

$\mathrm{S}=$ erro na medida (desvio padrão),

$\mathrm{k}=$ número de fatores experimentais no planejamento fatorial.

É importante salientar que por ser de valores médios, o erro nos efeitos é menor que o da medida. Também cabe ressaltar que o erro de uma média de uma amostragem é sempre menor que do individual de qualquer elemento da amostragem. 


\subsection{DETALHAHENTO DO PLANEJAMENTO FATORIAL}

Planejar experimentos é definir uma seqüência de coletas de dados experimentais para atingir certos objetivos. Dentre os métodos de planejamento experimental disponíveis na literatura, o planejamento fatorial é o mais indicado quando se deseja estudar os efeitos de duas ou mais variáveis de influência, sendo que em cada tentativa ou réplica, todas as combinações possíveis dos níveis de cada variável são investigadas (BARROS NETO et al., 1996).

De acordo com NEVES et al. (2002), este tipo de planejamento normalmente é representado por $b^{k}$, sendo que $k$ representa o número de fatores e " $b$ " o número de níveis escolhidos (NEVES et al., 2002). Para estes autores o caso mais simples de planejamento fatorial é aquele em que cada fator $k$ está presente em apenas dois níveis (experimento fatorial $2^{k}$ ), ou seja, em um experimento com $k$ fatores (ou variáveis) e dois níveis, são realizadas $2 \mathrm{x}$ $2 \times \ldots \times 2$ ( $k$ vezes) $=2^{k}$ observações da variável resposta. Esta representação mostra que, se em um planejamento forem escolhidos 2 diferentes níveis para 3 fatores $\left(2^{3}\right)$, o número de experimentos diferentes a serem realizados será 8.

Apesar dos planejamentos fatoriais do tipo $2^{k}$ serem mais comuns, e que com um número reduzido de níveis é praticamente impossível explorar de maneira completa uma grande região no espaço das variáveis (>4), estes ainda se mostram vantajosos, pois permitem verificar tendências importantes para a realização de investigações posteriores (NEVES et al., 2002). Neste tipo de planejamento fatorial, no qual os níveis são costumeiramente codificados com os sinais (+) e (-), a atribuição aos níveis superiores ou inferiores se dá de forma arbitrária e não interfere na realização dos experimentos ou interpretação dos resultados. Estes sinais também permitem esquematizar estas variáveis na forma de matrizes de planejamento, assim como determinar, por meio de cálculos, a influência das mesmas e das suas interações no sistema (BRASIL et al., 2007).

Além do exposto, cabe ressaltar que em um planejamento fatorial, as réplicas ou repetições de experimentos são de fundamental importância e servem para determinar o erro experimental na resposta em estudo e/ou a reprodutibilidade do esquema experimental utilizado (metodologia + equipamento).

Também é importante esclarecer que números de experimentos e número de condições experimentais são conceitos distintos, já que certo número de réplicas deve ser adicionado ao número de condições experimentais para fornecer o número de experimentos. Sendo assim, todos os ensaios e replicatas devem ser realizados de forma aleatória, visando evitar distorções estatísticas comprometedoras da qualidade dos resultados obtidos e dos efeitos calculados para as variáveis estudadas (GALDÁMEZ, 2002).

\subsection{VANTAGENS APRESENTADAS PELA UTILIZAÇÃO DO PLANEJAMENTO FATORIAL DE EXPERIMENTOS}

Dentre as diversas vantagens da utilização do planejamento fatorial, destacam-se as seguintes (BUTTON, 2005):

- redução do número de ensaios sem prejuízo da qualidade da informação;

- estudo simultâneo de diversas variáveis, separando seus efeitos;

- determinação da confiabilidade dos resultados;

- realização da pesquisa em etapas, num processo interativo de acréscimo de novos ensaios;

- seleção das variáveis que influenciam um processo com número reduzido de ensaios; 
- representação do processo estudado através de expressões matemáticas;

- elaboração de conclusões a partir de resultados qualitativos.

\section{EXEMPLO DE APLICAÇÃO PRÁTICA DO PLANEJAMENTO FATORIAL}

Com a finalidade de proporcionar um melhor entendimento das considerações acima descritas, será apresentado um exemplo de matriz de planejamento fatorial, na qual são levadas em consideração 2 variáveis quantitativas (concentração e tempo), e mostrados os resultados das duplicatas, assim como as suas médias e interações existentes.

É importante ressaltar que este exemplo trata de um estudo de otimização de variáveis operacionais relevantes, visando a fotopolimerização do metacrilato de metila (MMA) da cola acrílica comercial B20 (BERKEL), a qual é composta por um oligômero do MMA, por radiação UV.

Como a influência de cada variável é estudada entre dois limites, o desenho corresponde a um planejamento fatorial $2^{2}$, no qual apenas 4 ensaios são necessários para avaliar todas as combinações possíveis, porém, para verificar a tendência de linearidade e cálculo do erro experimental, um ponto central ensaiado em triplicata foi incluído neste estudo.

Cabe ressaltar que se fez necessário ter um conhecimento prévio do sistema em estudo para que fosse criteriosamente estabelecida a seleção de variáveis empregadas no estudo, assim como dos níveis em que estas foram ensaiadas, além da resposta utilizada no planejamento (a quantidade de polímero obtida, em \%, denominada de conversão).

Todos os ensaios foram realizados em escala de bancada, utilizando-se como solvente o tetracloreto de carbono P.A. (Merck). A fotopolimerização foi realizada dentro de uma câmara UV (254-400 nm, $9 \mathrm{~W})$. Como a cola acrílica utilizada apresentava em sua formulação um iniciador com identidade não revelada pelo fornecedor, uma adição complementar de fotoiniciador não foi realizada, para o presente estudo. O controle do processo de fotopolimerização (curva de conversão) foi realizado por determinações gravimétricas, aplicadas de acordo com procedimentos descritos na literatura (RODRIGUES; NEUMANN, 2003; FREGOLENTE, 2006).

\section{RESULTADOS E DISCUSSÃO}

Os resultados obtidos no experimento empregado como exemplo prático de planejamento fatorial encontram-se na Tabela 1, a seguir. 
TABELA 1 - PLANEJAMENTO FATORIAL $2^{2}$ PARA OTIMIZAÇÃO DAS CONDIÇÕES DE FOTOPOLIMERIZAÇÃO DE MMA COMERCIAL (BERKEL) POR UV.

[RESPOSTA: \% DE OBTENÇÃO DE POLI(METACRILATO DE METILA), PMMA, POR UV]

\begin{tabular}{|c|c|c|c|c|c|c|}
\hline \multicolumn{4}{|l|}{ VARIÁVEL } & Nível (-) & Nível (0) & Nível (+) \\
\hline \multicolumn{4}{|c|}{ Concentração $(C): \mathrm{m}_{\mathrm{MMA}} / \mathrm{v}_{\text {iniciador }}(\mathrm{mg} / \mathrm{mL})$} & $\mathrm{m} / 2$ & $\mathrm{~m} / 1,5$ & $\mathrm{~m} / 1$ \\
\hline \multicolumn{4}{|c|}{ Tempo de exposição ao UV $(t): \min$} & 0,5 & 1,0 & 1,5 \\
\hline \multirow{2}{*}{ EXPERIMENTO } & \multicolumn{3}{|c|}{ VARIÁVEIS } & \multicolumn{3}{|c|}{ RESPOSTA } \\
\hline & $\bar{c}$ & $\bar{t}$ & $C \times t$ & $\mathbf{R}_{\mathbf{1}}$ & $\mathbf{R}_{\mathbf{2}}$ & $\overline{\mathbf{R}}$ \\
\hline 1 & - & - & + & 21,36 & 24,71 & 23,04 \\
\hline 2 & + & - & - & 43,29 & 44,55 & 43,92 \\
\hline 3 & - & + & - & 20,99 & 21,14 & 21,07 \\
\hline 4 & + & + & + & 34,72 & 38,91 & 36,81 \\
\hline 5 & 0 & 0 & 0 & 28,46 & 29,88 & 29,17 \\
\hline 6 & 0 & 0 & 0 & 29,56 & 30,98 & 30,27 \\
\hline 7 & 0 & 0 & 0 & 30,66 & 32,08 & 31,37 \\
\hline
\end{tabular}

Efeitos principais: $C$ : $[(-23,04+43,92-21,07+36,81) / 2]=+18,31 \pm 1,51$

$t:[(-23,04-43,92+21,07+36,81) / 2]=-4,54 \pm 1,51$

Efeitos de segunda ordem: $C x t:[(+23,04-43,92-21,07+36,81) / 2]=-2,57 \pm 1,51$

De acordo com as informações contidas na Tabela 1, a única diferença entre os ensaios 1 e 2 está representada pela modificação da concentração de MMA. Desta forma, a diferença observada na resposta $(43,92-23,04=+20,88)$ indicou que a polimerização aumenta $20,88 \%$ quando a concentração de MMA $(C)$ muda do nível inferior (-) para o superior (+), mantendose fixo, no nível inferior, o tempo de exposição ao UV $(t)$. Uma análise similar permitiu calcular o efeito da concentração do MMA quando $t$ foi mantido fixo no nível superior (ensaios 3 e 4). Nesta análise, o efeito $(36,81-21,07=+15,74)$ indicou um aumento de $15,74 \%$ na quantidade, em \%, de polímero obtida, quando a concentração de MMA foi alterada do nível inferior (-) para o superior $(+)$. O efeito global apresentado pela variável $C$ foi obtido por meio da média aritmética dos efeitos supracitados $\{[(20,88+15,74) / 2]=+18,31]$.

Com cálculos similares aos descritos para $C$, foi possível verificar o efeito global para a variável $t(-4,54)$, o qual indicou que quando $t$ passa do nível inferior (-) para o superior $(+)$ a resposta monitorada sofre uma diminuição de 4,54\%. Estes resultados, associados ao desvio padrão apresentado nestes ensaios $( \pm 1,51)$, permitiram concluir que ambas variáveis influenciam a resposta monitorada, porém, a variável $C$, de forma significativa.

Se a intenção fosse maximizar esta resposta, as condições escolhidas deveriam corresponder aos níveis superiores de ambas variáveis (experimento 4), porém, observando os dados apresentados na Tabela 1, pode ser verificado que a melhor resposta foi dada para o experimento 2. A explicação para este fato se origina na pequena interação existente entre as variáveis $C$ e $t$.

A interpretação destes resultados pode ser facilitada com o auxílio da Figura 1, na qual estão representadas graficamente as respostas obtidas para os experimentos realizados como função das variáveis estudadas. Este tipo de representação (representação geométrica) é freqüentemente utilizado e tem como objetivo fornecer uma visão global de como as variáveis otimizadas atuam sobre a resposta do sistema químico em estudo. 


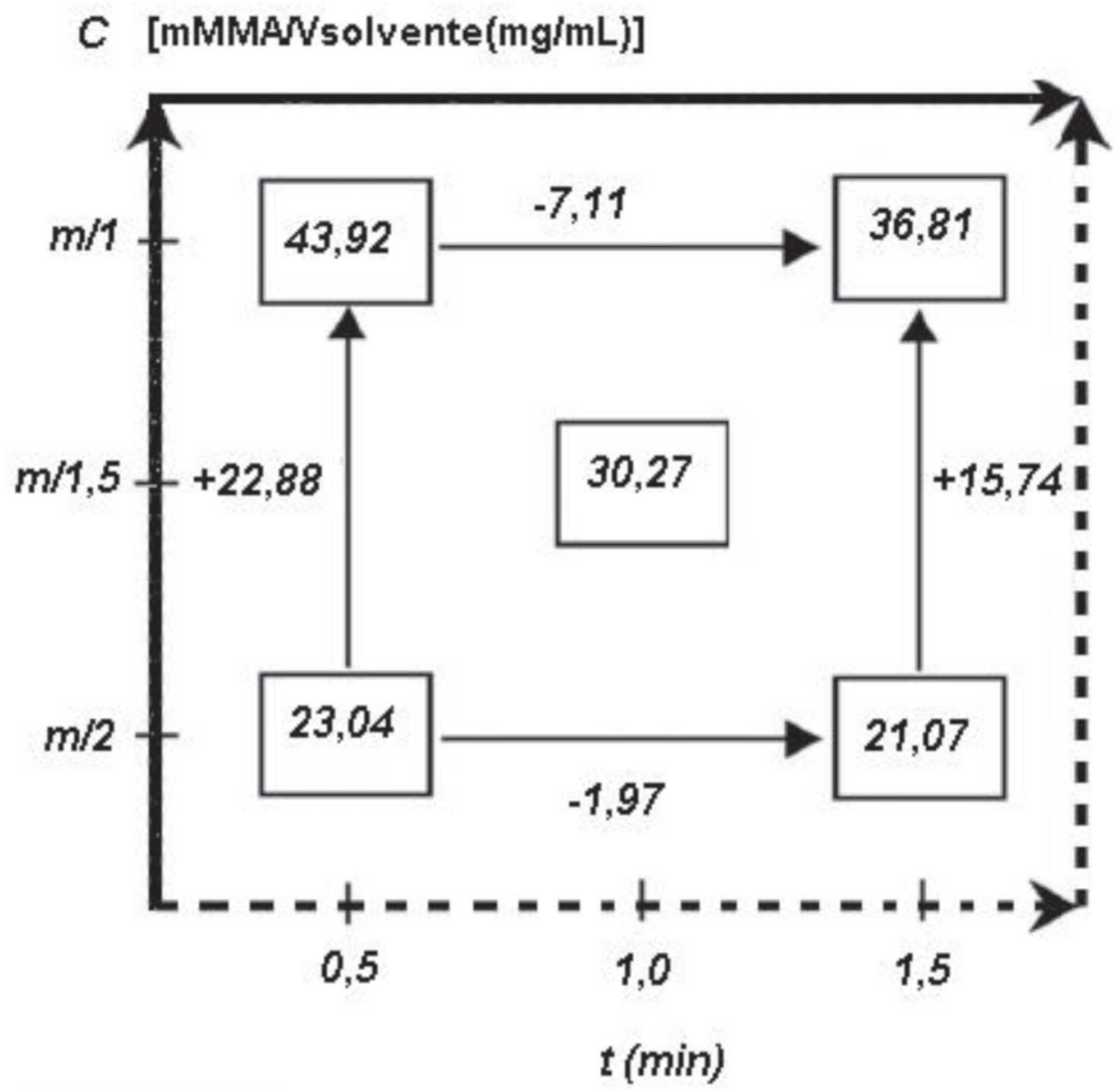

FIGURA 1 - REPRESENTAÇÃO GEOMÉTRICA DO PLANEJAMENTO FATORIAL $2^{2}$ DO SISTEMA $C \times t$ NA FOTOPOLIMERIZAÇÃO DO MMA COMERCIAL (BERKEL) POR RADIAÇÃO UV.

[RESPOSTA: \% DE OBTENÇÃO DE POLI(METACRILATO DE METILA), PMMA, POR UV]

$\mathrm{Na}$ representação geométrica (Figura 1), pode ser observado que o efeito da concentração de MMA, $C$, não é o mesmo para os níveis inferiores (-) e superiores (+) de $t$. Esta diferença também é percebida quando se avalia o efeito de $t$, e somente pode ser explicada em termos de interação entre variáveis. O valor numérico associado a esta interação (efeito de segunda ordem), foi calculado e encontra-se disponível na Tabela 1. Mesmo em situações de interação não tão significativa quanto esta, o sistema de planejamento fatorial demonstra o seu valor. Se um procedimento de otimização univariado tivesse sido executado (caminho das setas pontilhadas), as condições recomendadas seriam as do experimento 4, com uma eficiência de polimerização de $36,81 \%$. Em contrapartida, se o caminho indicado pelas setas não pontilhadas fosse escolhido, o valor otimizado real teria sido alcançado (experimento 2), com uma eficiência de polimerização de $43,92 \%$. Desta forma, a capacidade do sistema de planejamento fatorial em avaliar as interações possíveis foi demonstrada, permitindo eliminar o caráter aleatório associado à escolha do caminho univariado.

Pela análise dos valores dos efeitos (principal e de interação) e considerando o desvio padrão destes efeitos, pode-se concluir basicamente que as informações obtidas pelo cálculo dos efeitos principais indicam que a concentração tem um efeito positivo marcante $(+18,31)$ e que o tempo de exposição à radiação UV tem um efeito negativo pouco significativo $(-4,54)$. Pelos cálculos dos efeitos secundários nota-se que os efeitos da concentração de MMA e do 
tempo de exposição à radiação UV não podem ser interpretados separadamente, apesar do valor de interação entre eles ser praticamente desprezível $(-2,57)$, pelo fato de apresentar-se próximo ao valor do erro padrão.

Os resultados desta análise evidenciaram que o melhor rendimento de fotopolimerização do MMA deve ser obtido no experimento em que este oligômero se encontre na condição de maior concentração e menor tempo de exposição ao UV. Isto pode ser visualizado no diagrama de contorno inserido na Figura 2.

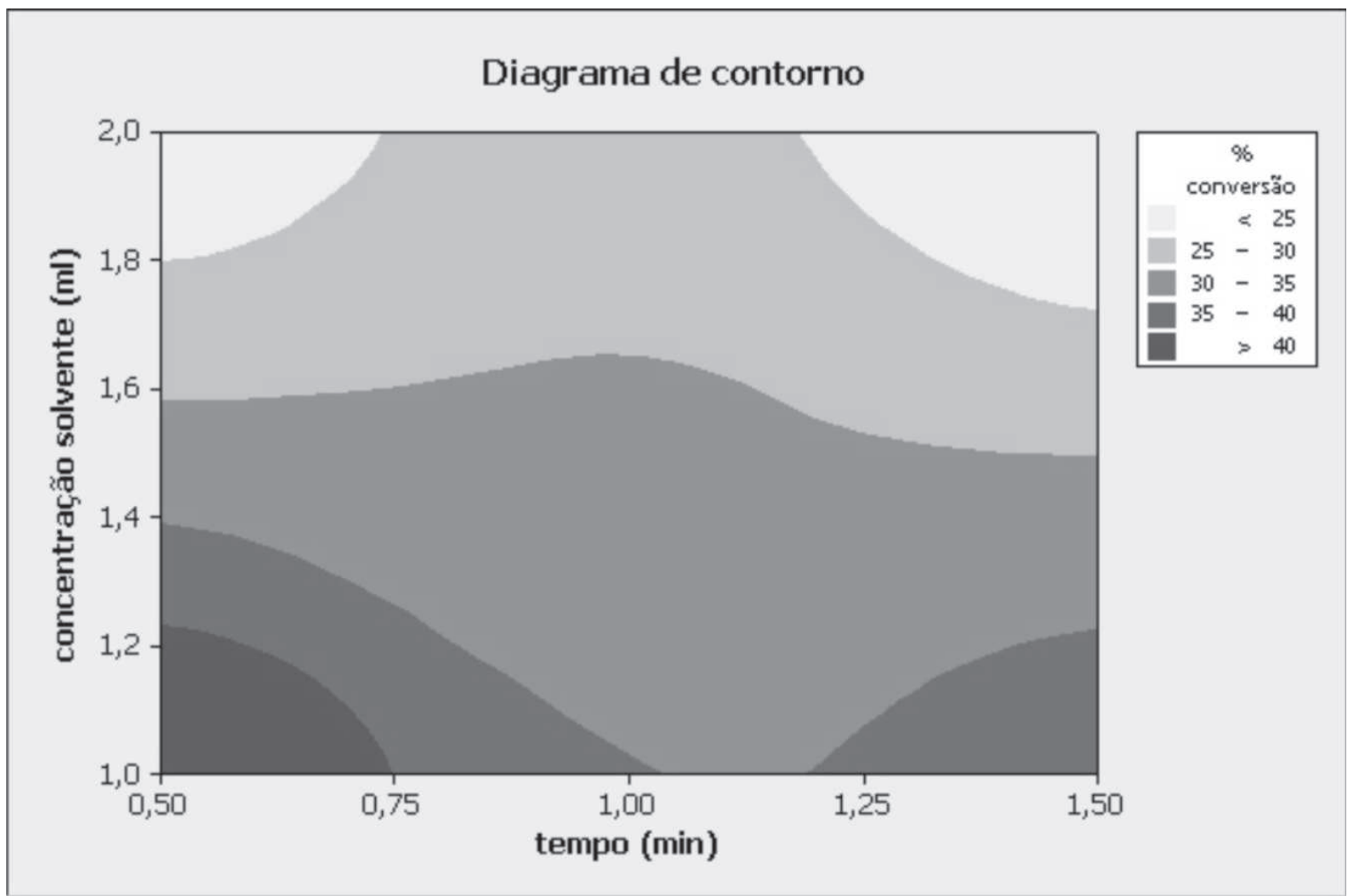

FIGURA 2 - DIAGRAMA DE CONTORNO DO SISTEMA $C \times t$ NA FOTOPOLIMERIZAÇÃO DO MMA COMERCIAL (BERKEL) POR RADIAÇÃO UV.

[RESPOSTA: \% DE OBTENÇÃO DE POLI (METACRILATO DE METILA), PMMA, POR UV]

Pelo exposto, pode-se perceber que o planejamento fatorial não determina valores ótimos em uma única etapa, porém este procedimento indica satisfatoriamente o caminho a ser tomado para que se possa atingir o objetivo proposto.

Também é importante ressaltar que embora o sistema permita evidenciar as interações entre as variáveis estudadas, a explicação deve ser fundamentada em argumentos técnicos relacionados com o processo em estudo. Sendo assim, no presente trabalho, o favorecimento do processo de fotopolimerização do MMA em um reduzido tempo de exposição ao UV pode ser explicado pela possível reação do oxigênio do ar com o iniciador em seu estado excitado trazendo-o para seu estado anterior, reduzindo temporariamente a formação de radicais livres. $\mathrm{O}$ radical formado pela fissão (quebra) do iniciador $\left(\mathrm{FI}^{*}\right)$ também pode ter reagido com $\mathrm{O}_{2}$ formando um radical peróxido, de baixa eficiência como iniciador de polimerização. Além disso, pode ainda ter reagido com o radical livre da cadeia em crescimento, reduzindo a eficiência da 
etapa de propagação (FREGOLENTE, 2006). Logo, o contato com oxigênio deve ser evitado ou minimizado com a utilização de reatores com colchão de nitrogênio ou outro gás inerte, ou pelo uso de filmes plásticos transparentes à radiação ultravioleta. Em contrapartida, selecionar fotoiniciadores com bandas de absorção em duas regiões distintas da zona espectral, atuando em faixas de absorção diferentes, uma maior e outra menor também pode ser considerada como outra forma de se lidar com este problema (TROCHMANN; BITTENCOURT, 2000).

\section{CONCLUSÕES}

O planejamento fatorial determina que fatores têm efeitos relevantes na resposta e, também, como o efeito de um fator varia com os níveis dos outros fatores. Além disso, permite estabelecer e quantificar as correlações entre os diferentes fatores. Diante do exposto, verificase que sem o uso de planejamentos fatoriais de experimentos, importantes interações entre fatores podem não são detectadas e a otimização máxima do sistema pode levar mais tempo para ser alcançada. Isto foi evidenciado neste trabalho, confirmando que os sistemas multivariados, fundamentados em planejamento fatorial de experimentos, permitem viabilizar o reconhecimento de condições de ótimo verdadeiro a partir de um reduzido número de experimentos.

\section{AGRADECIMENTOS}

Ao CNPq, pelo suporte financeiro.

À UFPR, pela estrutura laboratorial

À UTFPR, pela estrutura laboratorial.

\section{REFERÊNCIAS}

BARROS NETO, B.; SCARMINIO, I.S.; BRUNS, R. E. Planejamento e otimização de experimentos. 2ed. Campinas: Editora da UNICAMP, 1996. 299p.

BUTTON, S. T. METODOLOGIA PARA PLANEJAMENTO EXPERIMENTAL E ANÁLISE

DE RESULTADOS. 2005. http://www.fem.unicamp.br/ sergio1/pos-graduacao/IM317/ im317.htm. Acessado em maio de 2007.

BRASIL, J. L.; VAGHETTI, J. C. P.; SANTOS JR , B. R. A.; SIMON, N. M.; PAVAN, F. A.; DIAS, S. L. P.; LIMA, E. C. Planejamento estatístico de experimentos como uma ferramenta para otimização das condições de biossorção de $\mathrm{Cu}$ (II) em batelada utilizando-se casca de nozes pecã como biossorvente. Quimica Nova, v.30, n.3, p.548-553, 2007.

CHEMKEYS, www.chemkeys.com/bra/md/peeo_6/mdoeq_1/mdpf_3/ mdpf_3.htm, acessado em abril de 2007.

FREGOLENTE, P. B. L. Estudo da fotopolimerização de monômeros monofuncionais e multifuncionais para produção e recobrimento de lentes oftálmicas. 2006. $100 f$. Dissertação (Mestrado em Engenharia Química) - Departamento de Tecnologia de Polímeros, Universidade Estadual de Campinas, Campinas. 
GALDÁMEZ, E. V. C. Aplicação das técnicas de planejamento e análise de experimentos na melhoria da qualidade de um processo de fabricação de produtos plásticos. São Carlos, 2002. 133p. Dissertação (Mestrado em Engenharia de Produção), Escola de Engenharia de São Carlos, Universidade de São Paulo.

NEVES, C. F. C., SCHVARTZMAN, M. M. A. M.; J., E. Variables search technique applied to gas separation. Química Nova. v.25, n 2, p.327-329, 2002.

PEREIRA-FILHO, E. R., POPPI, R. J.; ARRUDA, M. A. Z. Employment of factorial design for optimization of pirolisys and atomization temperatures for $\mathrm{Al}, \mathrm{Cd}$, Mo and $\mathrm{Pb}$ determination by ETAAS. Química Nova. v. 25, n. 2, p.246-253, 2002.

PERALTA-ZAMORA, P.; MORAIS, J. L.; NAGATA, N. Por que otimização multivariada? Engenharia Sanitária e Ambiental. v.10, n.2, p.106-110, 2005.

RODRIGUES, M. R.; NEUMANN, M. G. Fotopolimerização: Princípios e Métodos. Polímeros: Ciência e Tecnologia. v.13, n.4, p.276-286, 2003.

TROCHMANN J. L. L., BITTENCOURT E., Uso de modelos de predição para índice de refração, temperatura de transição vítrea, comprimento e densidade de reticulação em copolímeros fotocuráveis. 2000. 100f. Dissertação (Mestrado em Engenharia Química) - Faculdade de Engenharia Química, Universidade Estadual de Campinas, Campinas. 